and proceeded immediately to the pharmacy, described lacking information (which they apparently overlooked). Treatment collection from pharmacies was acceptable, but sometimes pharmacy staff lacked knowledge of the OCP (despite training), causing delays and conversations which threatened patients' privacy - undermining the OCP's perceived benefits. For those routed to clinic (as opposed to choosing to attend), the OCP's anticipated benefits were also compromised. They described annoyance, anxiety, and did not always understand why a clinic visit was needed. The helpline was valued; users found it reassuring and informative.

Conclusion The OCP is a promising adjunct to traditional care, particularly when integrated into sexual health services. Critical points in users' journeys include the interface with pharmacies and clinics. Implementation issues in pharmacies need addressing. Management of users' expectations and tailored information may improve the experience when routed to clinic.

\section{O011 HITTING THE BULL'S-EYE: PARTNER NOTIFICATION REAL-TIME METRICS}

${ }^{1}$ Anatole Menon-Johansson*, 'Leigh Barlow. 'Guy's \& St Thomas' NHS Foundation Trust, London, England, UK; '2Sydney Medical School, Sydney, New South Wales, Australia

\subsection{6/sextrans-2016-052718.11}

Introduction Partner notification (PN) is a key but challenging service to deliver. The gold standard for PN confirmation is health care worker (HCW) verification. A cloud-based anonymous tool was developed to inform partners, support them to find a testing service and record when the HCW uses a unique code to reveal the sexually transmitted infection (STI), timing of $\mathrm{PN}$ initiation and closing the PN loop.

Objective To test the impact of the new tool on PN delivery. Methods A live pilot commenced on 27th January 2016 and analysis of all index patients using the PN tool over 49 days was performed using spreadsheet pivot tables and formulas.

Results A total of 259 index patients across nine providers with nine different STIs were analysed. These index patients declared 421 contactable contacts and 162 (38\%) were informed using the tool. A total of $96(59 \%)$ partners contacted opened the link embedded in their text message or email and 30 (31\%) were seen and tested by a HCW. A total of 13 STI testing centres received partners and the median (range) distance $\&$ time from PN initiation to HCW verification was $2.1(0.0-12.3)$ kilometres \& 63.8 (1-189.5) hours respectively

Discussion The PN tool has demonstrated that it is able to support partners to find a service and get tested expeditiously. The limited number of partners being informed is the rate limiting step and more work is required to develop strategies to enable effective $\mathrm{PN}$ initiation.

\section{UNDERSTANDING THE SEXUAL APPETITES OF MEN WHO HAVE SEX WITH MEN (MSM) TAKING PART IN A SEXUAL RISK REDUCTION INTERVENTION AFTER BEING PRESCRIBED POST EXPOSURE PROPHYLAXIS FOR HIV AFTER SEXUAL EXPOSURE (PEPSE)}

Alex Parr*, Carrie Llewellyn. Brighton \& Sussex Medical School, Brighton, UK
Background/introduction Risky sexual behaviours remain the drivers behind new HIV infections within MSM in the UK. Understanding reasons behind risk behaviour could inform HIV prevention strategies.

Aim(s)/objectives To document sexual appetite/libido among MSM taking PEPSE, and to examine the relations between libido and risk behaviours.

Methods Data were collected at enrolment, as part of an ongoing RCT evaluating a behavioural intervention to reduce HIV risk behaviour. Within this study, a 10 -item measure of libido was included (possible range of scores 10-40). This assessed how much thoughts and feelings about sex were considered disruptive.

Results 171 MSM responded (mean age 34.5, SD 9.1, range 1966 yrs). Mean (SD) libido score was 20.4 (7.7); median was 19 (range 30). Those with higher libido reported a higher number of sexual partners, both insertive $\left(r_{s}=0.298 ; p \leq 0.001\right.$; $\mathrm{n}=162)$ and receptive $\left(r_{s}=0.329 ; p \leq 0.001 ; \mathrm{n}=164\right)$, and inconsistent condom use, both insertive $\left(r_{s}=0.185 ; p=0.042\right.$; $\mathrm{n}=121)$ and receptive $\left(r_{s}=0.227 ; p=0.009 ; \mathrm{n}=132\right)$. Higher libido was associated with higher levels of loneliness $\left(r_{s}\right.$ $=0.401 ; p \leq 0.001 ; \mathrm{n}=165) ;$ reduced self-efficacy $\left(r_{s}=\right.$ $-0.230 ; p=0.003 ; \mathrm{n}=165)$, action planning $\left(r_{s}=-0.182 ; p\right.$ $=0.019 ; \mathrm{n}=164)$, intentions $\left(r_{s}=-0.163 ; p=0.036\right.$; $\mathrm{n}=165)$ and behavioural likelihood $\left(r_{s}=-0.228 ; p=0.003\right.$; $\mathrm{n}=165)$ of performing safer sex strategies. Loneliness was related to inconsistent condom use, both insertive $\left(r_{s}=0.191 ; p\right.$ $=0.021 ; \mathrm{n}=147)$ and receptive $\left(r_{s}=0.165 ; p=0.036\right.$; $\mathrm{n}=162)$, and a high number of sexual partners (receptive) $\left(r_{s}=\right.$ $0.164 ; p=0.033 ; \mathrm{n}=171$ ).

Discussion/conclusion Risky behaviours are related to stronger sexual appetites/libido and loneliness within this sample. The potential importance of libido and loneliness should be recognised within the context of future HIV prevention efforts.

\section{INVESTIGATING ATTITUDES TOWARDS HIV PRE-EXPOSURE PROPHYLAXIS (PREP). A QUESTIONNAIRE STUDY IN MEN WHO HAVE SEX WITH MEN ATTENDING SEXUAL HEALTH CLINICS}

${ }^{1}$ Lauren Bull*, ${ }^{1}$ Pavle Dimitrijevic, ${ }^{1}$ Alexander Scarborough, ${ }^{1}$ Sophie Beverley, ${ }^{1}$ Tristan Barber, ${ }^{2}$ lain Reeves, 'Sheena McCormack, 'Michael Rayment. 'Chelsea and Westminster Hospital, London, UK; ${ }^{2}$ Homerton Hospital, London, UK

\subsection{6/sextrans-2016-052718.13}

Background/introduction With the efficacy of HIV pre-exposure prophylaxis (PrEP) proven, provision of PrEP is currently being evaluated by commissioners. The question of who would wish to access PrEP, and where, is important in informing this process.

Aim(s)/objectives To establish potential users' attitudes towards, and experiences of, PrEP.

Methods Ethical approval was obtained to conduct a multicentre, prospective, anonymised questionnaire study of 1000 HIV negative MSM accessing sexual health clinics. Sexual behaviour, drug use, STI history and previous post exposure prophylaxis (PEP) use were collected. Opinions and attitudes towards PrEP and PrEP availability were assessed.

Results Of 386 analysed questionnaires the majority were British-born (203, 53\%), white $(300,78 \%)$ men. 345 (89\%) reported anal sex within the last month with 168 (43\%) and $139(36 \%)$ reporting unprotected insertive and receptive anal intercourse, respectively $(103,(26 \%)$ and $64,(17 \%)$ with 
multiple partners). 194 (50\%) had recently used recreational drugs (within 3 months; 34\% "Chemsex" substances). 157 (41\%) reported a recent STI (6 months). 223 (58\%) reported that they strongly believed they would benefit from PrEP. However, 42/223 (19\%) reported no condomless sex. Concerns around taking PrEP were cited by 76 (20\%). 167 (43\%) expressed a preference for daily PrEP; 139 (38\%) for coitallydriven. 311 (80\%) supported PrEP delivery by sexual health clinics to MSM, and $233(60 \%)$ to any-one who requests it. 112 (29\%) agreed a prescription charge was appropriate. 17 respondents (4\%) reported having already taken PrEP: 35\% using medication acquired as PEP, and 30\% acquiring PrEP privately. 7/17 (41\%) reported decreased condom since commencing PrEP.

Discussion/conclusion This comprehensive questionnaire study demonstrates a high willingness to use PrEP in a cohort of atrisk MSM. These data should inform the commissioning process of this efficacious biological intervention.

\section{ESTABLISHMENT OF A MONITORING SERVICE FOR MEN WHO HAVE SEX WITH MEN (MSM) TAKING GENERIC CO-FORMULATED TENOFOVIR DISOPROXIL FUMARATE (TDF)/EMTRICITABINE (FTC) AS PRE-EXPOSURE PROPHYLAXIS (PREP) AGAINST HIV INFECTION}

${ }^{1}$ Nneka Nwokolo, ${ }^{2}$ Xinzhu Wang, ${ }^{1,2}$ Marta Boffito, ${ }^{2}$ Myra McClure, ${ }^{1}$ Tara Suchak* ${ }^{1}$ Gary Whitlock. '56 Dean Street, Chelsea \& Westminster Hospital, London, UK; ${ }^{2}$ Imperial College, London, UK

10.1136/sextrans-2016-052718.14

Background/introduction Truvada ${ }^{\circledR}$ (TDF/FTC) PrEP taken daily or intermittently reduces HIV acquisition by over $86 \%$. However, PrEP is only available privately in the UK, costing upwards of $£ 400$ for 30 tablets. Online generic TDF/FTC is significantly cheaper at $£ 35$ - $£ 50$ for 30 tablets. There are, however, authenticity concerns about online medicines. Additionally, HIV infection should be excluded in individuals taking PrEP and baseline assessments of hepatitis B and renal function performed which may not occur with online PrEP.

Since February 2016, we have provided assessment and therapeutic drug monitoring to individuals on generic TDF/FTC to ensure safety and medication integrity.

Aim(s)/objectives To review characteristics of individuals taking generic TDF/FTC.

Methods Service evaluation of individuals taking generic TDF/ FTC attending a London sexual health service. Data on the first 44 patients were collected: demographics, HIV and renal function testing, hepatitis B status, baseline STIs, regimen, source of PrEP.

Results All MSM; mean age 41 years (28-73); 77\% White; 33/ $44(75 \%)$ on PrEP at time of attendance; all HIV antibody negative prior to commencement. Mean eGFR $81.5 \mathrm{ml} / \mathrm{min}, 65 \%$ had documented hepatitis B immunity. One STI (syphilis) was identified at baseline. 93\% were taking daily PrEP and $86 \%$ obtained Cipla manufactured Tenvir-EM ${ }^{\circledR}$ from United Pharmacies. Tenofovir and FTC levels were measured in 18/44 (41\%), all results demonstrating presence of adequate active compound. Discussion/conclusion Numbers of individuals requiring monitoring on generic TDF/FTC are increasing. It is reassuring that so far, drug levels suggest appropriate quantities of tenofovir and FTC in Tenvir-EM ${ }^{\circledR}$; however, more data are needed.

\section{RENAL FUNCTION AT BASELINE AND MONTH 1 IN THE PROUD STUDY, A PRAGMATIC OPEN LABEL RANDOMISED TRIAL OF TRUVADA AS PRE-EXPOSURE PROPHYLAXIS}

${ }^{1}$ lain Reeves*, ${ }^{2}$ Ellen White, ${ }^{2}$ Elizabeth Brodnicki, ${ }^{2}$ David Dunn, ${ }^{2}$ Sheena McCormack, ${ }^{3}$ Tristan Barber, ${ }^{3}$ Ann Sullivan, ${ }^{5}$ Charles Lacey, ${ }^{4}$ Hannah Alexander. ${ }^{1}$ Homerton University Hospital, London, UK; ${ }^{2}$ MRC CTU, London, UK; ${ }^{3}$ Chelsea and Westminster NHS Trust, London, UK; ${ }^{4}$ Kings College Hospital, London, UK; ${ }^{5}$ Hull York Medical School, York, UK

\subsection{6/sextrans-2016-052718.15}

Background/introduction Quarterly monitoring of creatinine is likely to be recommended by WHO for those on PrEP, even though there were no significant differences in creatinine in placebo-controlled trials. Establishing the appropriate level of monitoring of PrEP is important.

Methods PROUD is an open-label, randomised trial of Truvada as PrEP in MSM. HIV serology and serum creatinine was done at PrEP baseline ('start'). Clinics were advised to collect creatinine or urinary protein-creatinine ratio (UPCR) if there was $\geq 1+$ protein on urinalysis at the month 1 visit (m1). Here we present the renal monitoring results at "start" and $\mathrm{m} 1$ with eGFR $\left(\mathrm{ml} / \mathrm{min} / 1.73 \mathrm{~m}^{2}\right)$ calculated by the CKD-EPI equation.

Results 445 (93\%) of 481 had baseline creatinine, 13 (3\%) had UPCR, and $23(5 \%)$ neither. The median eGFR was 106. Only one was $<60($ eGFR $=49)$, probably due to dietary creatinine supplementation. 260 (59\%) of 443 had a $\mathrm{m} 1$ creatinine, creating 246 paired results. On average, eGFR was 1.50 lower at $\mathrm{m} 1$. Seven (4\%) of 194 with eGFR >90 dropped 20\%, one to 59. He stopped PrEP and did not attend thereafter. Of the 7 , none had abnormal urinalysis; 4 had UPCR - all normal. 41 (79\%) of 52 with eGFR 60-90 at baseline remained at this level, the remainder increased to $>90$.

Discussion/conclusion The mean change in eGFR at month 1 is not clinically significant. Excepting one individual who could not be further evaluated, there were no clinically meaningful changes at $\mathrm{m} 1$. Further work will explore the relationships between eGFR and proteinuria.

\section{CHEMSEX RELATED ADMISSIONS TO A CITY CENTRE HOSPITAL}

${ }^{1}$ Chris Ward*, 'Debbie Thomas, ${ }^{2,1}$ Terri Anderson, ${ }^{1}$ Rebecca Evans, ${ }^{1}$ Orla McQuillan. ${ }^{1}$ Central Manchester University Hospitals NHS Foundation Trust, Manchester, UK; ${ }^{2}$ Addiction Dependency Solutions, Manchester, UK

\subsection{6/sextrans-2016-052718.16}

Background/introduction Recreational drug use (RDU), particularly the chemsex drugs mephedrone, crystal methamphetamine and gamma-hydroxybutyric acid (GHB) are associated with significant harms. Occasionally this has led to hospital admission with significant morbidity and mortality.

Aim(s)/objectives To review inpatient admissions from a large HIV service and look at RDU associations.

Methods A prospective analysis of admissions to an HIV inpatient service between April 2015 and March 2016 was conducted. Information was collected on demographics, admission details, complications and drug use.

Results From 194 admissions there were 19 (9.8\%) related to RDU. Median age was 33.5 (range 23-65). All were male and $18(94.7 \%)$ were men who have sex with men (MSM). 4 (21.1\%) were Hepatitis C co-infected. 5 (26.3\%) patients took 